\title{
Hypertensive Urgency: The Role of Clinical Pharmacist in Identifying and Managing Drug Therapy Problems: The Case of Ambo Hospital Medical Ward, Ethiopia
}

Jimma Likisa Lenjisa*, Minyahil Alebachew Woldu, Gobeze Temesgen, Gurmu Tesfaye, Awoke Shiferaw, Anchalu Yadeta and Assefa Maraba

Ambo University, College of Medicine and Health Sciences, School of Pharmacy, Department of Clinical Pharmacy, Ethiopia

\begin{abstract}
Worldwide the role of clinical pharmacists is becoming valuable and expanding in an alarming rate especially in developed countries. However, it is at its infancy stage in low income countries like Ethiopia. Therefore, this particular case is meant to show how much clinical pharmacists are important in the identification and management of drug therapy related problems among hospitalized patients. Accordingly, it was found that clinical pharmacists were able to diagnosis drug related problems during the management of hypertensive urgencies and provide evidence based recommendation to address the problems identified. We recommend the need for more number of clinical pharmacists in the area as well as devotion from the policy makers in upgrading their knowledge.
\end{abstract}

Keywords: Clinical pharmacy; Drug therapy problem; Hypertensive urgency; Ambo hospital

\section{Introduction to Clinical Pharmacy Services}

Clinical pharmacy had begun its journey in United States of America in 1960s. From that time onwards, it has been broadening its horizon to different parts of the world though with very slow pace. However, secondary to the year 2000 WHO recommendation, expanding and adopting clinical pharmacy role was a must than a right as drug related problems are causing tremendous harm to the life and quality of life of the world population [1]. For this reason, pharmacist today are drug therapy experts not only drug experts, responsible providers of drug therapy not only pushing a pill behind the counter, and evidence based medicine practitioners as they are providing unbiased drug information for the patients, other health care professionals and community at large. Because of their deepest knowledge in pharmacology, therapeutics, pharmacoeconomics, pharmacoepidemiology and drug information researches, clinical pharmacists are the best weapons to fight the sequels being imposed by drug therapy problems during management of diseases [1].

This is especially true in developing world where the magnitude of harms caused by drug therapy problems has been deep rooted. These problems include but not limited to adverse drug reactions, prescribing drugs with no indication, inappropriately treating identified disease, prescribing drugs regardless of cost consideration and potential risk to special population like paediatrics, geriatrics and pregnant women. as well as changing medication and prescribing medications without considering medication history and medication taking behaviours of the patients are also within the spectrum of drug therapy problems [1].

Moreover, Drug therapy problems are significant cause of morbidity, hospital admission and mortality. But for long periods of time in history they had been neglected by policy makers of many countries in the world while there importance as a cause of child and maternal mortality is as significant as that of mortality caused by HIV, TB, Malaria and pregnancy related complications. Now a day, by realizing these facts $\mathrm{WHO}$ and the world community at large are promoting clinical pharmacy service to implement in health care system of one's country as well as to deliver clinical pharmacy based curriculum in their universities. To this end, Ethiopia is one of the countries which are being benefited from the service even if the shortage of manpower in this regard is the worst. Therefore, the objective of this case report is to show how clinical pharmacists are providing direct patient care being drug therapy expert not only drug expert in one of the largest hospital in Ethiopia called Ambo Hospital considering prototype case on management of hypertensive urgency in medical ward [1].

\section{Scenario}

\section{Patient information}

Card number: 026094

Admission date: 16/03/2014

Sex: female

Age: 56 years

Ward: Medical ward

Bed number: $5 / 3$

\section{History}

Chief complaint: Dry cough associated with chest pain for 02 days.

History of present illness: Mrs.T. T isa previously healthy 56 years old patient presented to female medical ward on 16/03/2014 with dry cough of two days duration. The cough was associated with chest pain. She has high grade fever and shortness of breath.

Past medical history: No History of hypertension, tuberculosis, diabetes mellitus (DM) and other chronic diseases

*Corresponding author: Likisa JL, Ambo University, College Of Medicine and Health Sciences, School of Pharmacy, Department of Clinical Pharmacy, Ethiopia, Tel: 251-917-305-585; E-mail: jimmapharm@gmail.com

Received April 14, 2013; Accepted May 22, 2014; Published May 25, 2014

Citation: Lenjisa JL, Woldu MA, Temesgen G, Tesfaye G, Shiferaw A, et al (2014) Hypertensive Urgency: The Role of Clinical Pharmacist in Identifying and Managing Drug Therapy Problems: The Case of Ambo Hospital Medical Ward, Ethiopia. J Bioanal Biomed 6: 015-018. doi:10.4172/1948-593X.1000102

Copyright: ( 2014 Lenjisa JL, et al. This is an open-access article distributed under the terms of the Creative Commons Attribution License, which permits unrestricted use, distribution, and reproduction in any medium, provided the original author and source are credited. 
Citation: Lenjisa JL, Woldu MA, Temesgen G, Tesfaye G, Shiferaw A, et al. (2014) Hypertensive Urgency: The Role of Clinical Pharmacist in Identifying and Managing Drug Therapy Problems: The Case of Ambo Hospital Medical Ward, Ethiopia. J Bioanal Biomed 6: 015-018. doi:10.4172/1948-593X.1000102

Family history: She has no family history of hypertension (HTN), and DM

Social history: She didn't chew chat, didn't smoke and drinks 3 or more cup of coffee per day. No history of smoking and any other social drug use. She is married and living with husband and children.

Medication history: Denied taking any home or prescription or over the counter (OTC) medication.

History of allergy: No known drug allergy

\section{Physical examination}

Vital signs on admission:

- PR: 70, RR:40, BP:190/100

- Cardiovascular system (CVS): hear sound 1 (S1) and heart sound 2 (S2) well heard, there is no murmur and gallop.

- Respiratory system (RS): decreased air entry on the posterior $2 / 3$ of the chest

- Abdomen: no palpable organs

- No abnormality was detected on other systems

\section{Laboratory investigations}

CBC: RBC---- $4.64 \times 10^{3}$ cells $/ \mathrm{mm}^{3}$

Hct: $39.3 \%$

ESR: $40 \mathrm{mg} / \mathrm{hr}$

Hgb: $12.3 \mathrm{~g} / \mathrm{dl}$

MCHC: $31 \%$

WBC: $4.7 \mathrm{X} 10^{3}$ cells $/ \mathrm{mm}^{3}$

Neutrophil: $41.1 \%$

PLT: $292 \times 10^{3}$ cells $/ \mathrm{mm}^{3}$

EOSINOPHIL: $14.3 \%$

Lymphocytes: $46.5 \%$

Chest X-ray (CXR): ordered but not found

Renal function test (RFT): ordered but not found

Diagnosis:

- Hypertensive Urgency

- Community Acquired Pneumonia (not severe)

\section{Medications on admission}

- Cetriaxone $1 \mathrm{~g}$ through vein (iv) twice daily (bid)

- Doxycycline $100 \mathrm{mg}$ per oral( po) twice daily

- Hydralazine $5 \mathrm{mg}$ iv every 20 min until $\mathrm{BP}<160 / 100 \mathrm{mmgh}$

- Nifedipine $20 \mathrm{mg}$ po bid

\section{Current Medications (from 17/03/2014 to discharge)}

- Cetriaxone 1g iv bid

- Doxycycline $100 \mathrm{mg}$ po bid

- Hydralazine $5 \mathrm{mg}$ iv every 20 min until $\mathrm{BP}<160 / 100 \mathrm{mmgh}$
- Nifedipine $20 \mathrm{mg}$ po bid

- Hydrochlorothiazide 25mg po/day

- Enalapril $5 \mathrm{mg}$ po bid

\section{Discharge Medications}

- Nifedipine (extended release) $20 \mathrm{mg}$ po bid $01 \mathrm{month}$

- Hydrochlorothiazide $25 \mathrm{mg}$ po/day 01 month

- Enalapril $5 \mathrm{mg}$ po bid for $01 \mathrm{month}$

- Amoxicillin $500 \mathrm{mg}$ potid( three times daily) for 7 days

Blood Pressure (BP) Chart

\begin{tabular}{|l|l|l|l|l|l|l|l|}
\hline Variables & & & & & & \\
\hline Date & $16 / 3 / 014$ & $17 / 3 / 014$ & $18 / 3 / 014$ & $19 / 3 / 014$ & $20 / 3 / 014$ & $21 / 3 / 014$ & $22 / 3 / 014$ \\
\hline Time & $1: 00 \mathrm{pm}$ & $7: 00 \mathrm{am}$ & $12: 00 \mathrm{am}$ & $12: 00 \mathrm{am}$ & $12: 00 \mathrm{am}$ & $12: 00 \mathrm{am}$ & $4: 00 \mathrm{pm}$ \\
\hline BP & $190 / 100$ & $140 / 80$ & $190 / 100$ & $170 / 100$ & $170 / 100$ & $160 / 100$ & $150 / 90$ \\
\hline
\end{tabular}

\section{Clinical questions}

1. Was thepatient's hypertensive urgency being treated with the most effective, appropriate and safe drugs as per the recent evidence based guidelines?

2. Was the choice of drug therapy for management of hypertensive urgency for the patient most economical?

3. Was choice of discharge medications appropriate?

4. Is management of non-severe community acquired pneumonia appropriate?

5. Was there a need to shift IV antibiotics to PO as patient stabilized?

6. Should amoxicillinbe included in discharge medications of the patient?

\section{Clinical pharmacists' recommendation}

1. Hypertensive urgency was not managed according to the recent evidence based guidelines. BP lowering in such patients (newly diagnosed patient who has no history of previous treatment) should not be urgent with IV medication like hydralazine. Recommended medications are oral captopril, labetalol etc...as discussed below. The intervention shouldn't be aggressive like this as the patient may develop extra complication.

2. The intervention given to the patient was not really considering cost of both medication and harm to the patients as a result of unnecessary overprescribing of antihypertensive drugs.

3. There is no need to discharge the patient with three antihypertensive drugs while even one is sufficient to control $\mathrm{BP}$ in such patient. If not one, two antihypertensive one of which is thiazide diuretics is appropriate intervention to minimize the risk of future drug therapy problems like adverse drug reactions (ADRs).

4. According to standard text books and recent guidelines, non severe form of community acquired pneumonia should not 
Citation: Lenjisa JL, Woldu MA, Temesgen G, Tesfaye G, Shiferaw A, et al. (2014) Hypertensive Urgency: The Role of Clinical Pharmacist in Identifying and Managing Drug Therapy Problems: The Case of Ambo Hospital Medical Ward, Ethiopia. J Bioanal Biomed 6: 015-018. doi:10.4172/1948-593X.1000102

be managed with combination of antibiotics. In this regard, macrolides are drugs of choice since the patient didn't have any history of antibiotics use in the last three months before admission. Tetracyclines like doxycycline are appropriate alternatives if we don't have macrolide antibiotics like clarithromycin [2-4].

5. In general IV to PO shift is the best way to utilize antibiotics in hospitals provided that the patients fulfilled the criteria to do so for several reasons; avoids injection site complications, minimize cost and reduce hospital stay.

6. Amoxicillin was unnecessarily included in discharge medications as the patients were being treated with full course of both doxycycline and ceftriaxone.

\section{Overview of Hypertensive Urgency and Its Managements}

\section{The Evidences for Clinical Pharmacists' Recommendations}

Hypertensive urgencies are characterized by the presence of very elevated $\mathrm{BP}$ - greater than $180 / 120 \mathrm{~mm} \mathrm{Hg}$. Urgencies are not associated with acute or immediately progressing target organ injury unlike hypertensive emergencies. Examples of acute target organ injury include encephalopathy, intracranial haemorrhage, and acute left ventricular failure with pulmonary edema, dissecting aortic aneurysm, unstable angina, and eclampsia or severe hypertension during pregnancy. Because there is no consensus regarding the cut off value of blood pressure for defining hypertensive urgencies, diagnosis should be individualized after taking into account several factors, such as age, sex, the presence of chronic hypertension (and use of antihypertensive drugs), and the presence of co morbidities [2,3].

Acute hypertension is not uncommon in the emergency room or acute care setting and can have important consequences on various organs. In this regard, it has been reported that end-organ injury has been reported in $19-81 \%$ of patients with acute severe hypertension. In a recent American survey of patients with severe acute hypertension (defined as systolic arterial pressure [SAP] $>180 \mathrm{mmHg}$ and/or diastolic arterial pressure $[\mathrm{DAP}]>110 \mathrm{mmHg}$ ) requiring hospitalization, new or worsening end-organ dysfunction was observed in $59 \%$ of subjects; the 90 -day mortality rate was $11 \%$. More than one third of patients discharged home were re-hospitalized at least once within 90 days, $29 \%$ for acute severe hypertension $[3,4]$.

\section{What causes hypertensive urgency?}

Most patients presenting with hypertensive urgency have been previously diagnosed with hypertension. Severe BP elevations may result from inadequate control on or poor adherence to current antihypertensive drug regimens. Another cause for patients reaching hypertensive urgency or emergency is previous inaccurate BP measurements that underestimate or do not detect increased BP at all (e.g. Poor patient technique for self-monitoring) [4-6].

- A patient's BP can also be elevated by other factors, including:

- Drugs like attention deficit hyperactivity disorders(ADHD) medications (e.g. methylphenidate), antidepressants (e.g. venlafaxine, buproprion, desipramine), calcineurin inhibitors (e.g. cyclosporine, tacrolimus), corticosteroids, estrogens, midodrine, non-steriodal anti inflammatory drugs (NSAIDs) (e.g. aspirin( ASA), ibuprofen, naproxen, diclofenac, celecoxib), testosterone, triptans, decongestants (e.g. pseudoephedrine, phenylephrine), NSAIDs (ibuprofen, naproxen), topical ASA or diclofenac

- Herbal: black licorice root, ginkgo biloba, St. John's Wort. Consider potential for undeclared ingredients/adulterants.

Recreational: stimulants (e.g. amphetamines like crystal meth or ecstasy), anabolic steroids, caffeine, cocaine, phencyclidine.

- Energy drinks containing taurine, guarana root, yerba mate, glucuronolactone, etc.

- Lifestyle: High salt diet, excessive alcohol use.

- Comorbid Conditions: Thyroid storm, trauma, renovascular disease, acute ischemic stroke or adrenal dysfunction

- Some population groups are more likely to experience hypertensive urgency

Elderly; African Americans; Men > Women [7,8].

\section{How Should Hypertensive Urgency be Managed?}

A common error with treating hypertensive urgency is initiating overly aggressive antihypertensive therapy. Hypertensive urgencies are ideally managed by adjusting maintenance therapy by adding a new antihypertensive and/or increasing the dose of a present medication. This is the preferred approach to these patients as it provides a more gradual reduction in BP. Very rapid reductions in BP to goal values should be discouraged because of potential risks [2-8].

Because auto regulation of blood flow in patients with hypertension occurs at a much higher range of pressure than in normotensive persons, the inherent risks of reducing BP too precipitously include cerebrovascular accidents, myocardial infarction (MI), and acute kidney failure. Hypertensive urgency requires BP reductions with oral antihypertensive agents to stage 1 values over a period of several hours to several days. All patients with hypertensive urgency should be revaluated within 7 days (preferably after 1 to 3 days) [3,5].

Overall Goal of Management: reduce SBP by roughly 25\% over 24-48 hours. More conservative BP lowering reduces the risk of potential adverse effects (i.e. perfusion complications worsening incidence of MI, stroke, and death) associated with more aggressive BP lowering. All treatment strategies should consider the patient's co morbidities and risk of adverse events.

1. All patients should be provided with a quiet room to rest. This will be adequate for some \& can lead to a fall in BP of $\geq 10-20$ mmHg.

2. Tilt the head of the bed 15 degrees up. This may also help to decrease BP.

3. If BP remains above $180 / 100 \mathrm{mmHg}$ for $\geq 3$ hours, consider anti-hypertensives.

4. General drug treatment options differ depending on whether the patient was previously diagnosed with \& treated for hypertension [6-8].

\section{Previously treated hypertension}

Trying one the following may be appropriate interventions (in no particular order):

$\checkmark$ Increase the dose of current antihypertensive medications

$\checkmark$ Add another antihypertensive agent from another class 
Citation: Lenjisa JL, Woldu MA, Temesgen G, Tesfaye G, Shiferaw A, et al. (2014) Hypertensive Urgency: The Role of Clinical Pharmacist in Identifying and Managing Drug Therapy Problems: The Case of Ambo Hospital Medical Ward, Ethiopia. J Bioanal Biomed 6: 015-018. doi:10.4172/1948-593X.1000102

$\checkmark$ Restart/resume medications in non-adherent patients

\section{Untreated hypertension}

Choice of treatment depends on patient and the setting (e.g. emergency room versus doctor's office)

$\checkmark$ Temporary use of fast acting oral anti-hypertensives (e.g. clonidine, labetalol, captopril) may be desirable to gradually reduce blood pressure over several hours, depending on the condition of the patient. Oral captopril is one of the agents of choice and can be used in doses of 25 to $50 \mathrm{mg}$ at 1- to 2-hour intervals. However, Oral or sublingual immediate-release nifedipine for acute BP lowering is dangerous. This approach produces a rapid reduction in $\mathrm{BP}$.

$\checkmark$ Reduction of blood pressure over 24-48 hours with longer acting agents (e.g. ramipril $10 \mathrm{mg}$, metoprololextended release (XL) $100 \mathrm{mg}$, or nifedipine XL $30 \mathrm{mg}$ )

$\checkmark$ After a few hours of observation, and roughly $20-30 \mathrm{mmHg}$ reduction in $\mathrm{BP}$, the patient should be prescribed a longer acting agent in accordance with treatment of essential hypertension (i.e. ACE-I/ARB, thiazide diuretic, calcium channel blocker). The choice of agent should consideration for what is most appropriate long term including co morbidities [6,7].

What are the Follow-up and Monitoring Parameters for Hypertensive Urgency?

- The patient should be observed for a few hours to confirm that they are stable/improving and asymptomatic.

- Once stable, the patient can be sent home with close follow-up (every 1-2 days) involving:

- Evaluation for signs of hypertension or hypotension

- Attainment of BP goals with the help of antihypertensive and lifestyle interventions (e.g. low salt diet, increased physical activity) BP goals may vary depending on the patient and any existing co morbid conditions.

- Assessment of medication adherence (consider simplified dosing schedules, and adherence aides, if appropriate)

If there are concerns about patient compliance to follow up, especially if there is high risk of cerebrovascular or cardiovascular disease, hospital admission may be warranted for initial management [5-8].

Follow up evaluation (patient monitoring) for the above case

\section{Monitoring parameters for efficacy}

Frequent monitoring of BP for the first two or three months.

\section{Monitoring parameters for safety}

Regularly monitoring renal function, blood sugar, uric acid analysis, BP for signs of hypotension and monitoring patients for signs like peripheral edema due to nifidepine.

\section{Patient education points addressed by Clinical pharmacists for this case}

- Avoid any sorts of stress

- Avoid the use of herbal remedies of unstandardized use
- Avoid the use of OTC medications and any drugs of social value.

- Reduce salt intake and moderation of alcohol intake like 2 drinks per day.

- Report to the nearby health institutions or health professionals in case she faces any sign and symptoms of ADRs which is already told to her.

- Importance of adherence to her medication. Recommendations to policy makers and hospital
administrators

In Ambo Hospital, currently, there are only two clinical pharmacists working as full time employee. All clinical specialities in the hospital are being run by these two individuals who are obviously difficult to manage every case effectively. They are not attending night shift which increase the probability of not involved in admission medication of some patients which is the main problem in this hospital. Hence, more number of clinical pharmacists considering all clinical speciality wards and night shift need to be recruited to avoid the big problems related to drug therapy observed in this prototype real patient case. In Ethiopia there are hospitals which even don't have one clinical pharmacist. Therefore, in general, there should be wider implementation of the clinical pharmacy services in this country in order to minimize the harm being caused by different types of drug therapy problems which are as important as addressing the problems of HIV/AIDS, malaria, $\mathrm{TB}$, pregnancy and child birth related complications and mortality.

\section{References}

1. WHO (2006) Developing Pharmacy practice: a focus on patient care.

2. Joseeph JS, Eric JM Pharmacotherapy: a pathophysiologic approach Hypertension. Hypertensive Urgencies and Emergencies. (7th edn) 139-171.

3. James PA, Oparil S, Carter BL, Cushman WC, Dennison-Himmelfarb C, et al. (2014) 2014 evidence-based guideline for the management of high blood pressure in adults: report from the panel members appointed to the Eighth Joint National Committee (JNC 8). JAMA 311: 507-520.

4. Salgado DR, Silva E, Vincent JL (2013) Control of hypertension in the critically ill: a pathophysiological approach. Ann Intensive Care 3: 17.

5. http://www.rxfiles.ca/rxfiles/modules/druginfoindex/druginfo.aspx

6. Padwal R, Poirier L, Quinn R (2013) The 2013 Canadian Hypertension Education Program (CHEP) recommendations.

7. http://www.uptodate.com/contents/management-of-severe-asymptomatichypertension-hypertensive-urgencies-in-adults

8. Dan perri. $5^{\text {th }}$ McMaster University Review Course on Internal medicine Management of hypertensive urgency and emergency. JNC 7, JAMA 2003; 289: $2560-2572$. 